\title{
Broadband terahertz modulators using self-gated graphene capacitors
}

\author{
Nurbek Kakenov, ${ }^{1}$ Osman Balci, ${ }^{1}$ Emre O. Polat, ${ }^{1}$ Hakan Altan, ${ }^{2,3}$ and Coskun Kocabas ${ }^{1, *}$ \\ ${ }^{1}$ Department of Physics, Bilkent University, 06800 Ankara, Turkey \\ ${ }^{2}$ Department of Physics, Middle East Technical University, 06531 Ankara, Turkey \\ ${ }^{3}$ e-mail: haltan@metu.edu.tr \\ *Corresponding author: ckocabas@fen.bilkent.edu.tr
}

Received 8 May 2015; revised 15 July 2015; accepted 19 July 2015; posted 20 July 2015 (Doc. ID 240535); published 13 August 2015

\begin{abstract}
We demonstrate a terahertz intensity modulator using a graphene supercapacitor which consists of two large-area graphene electrodes and an electrolyte medium. The mutual electrolyte gating between the graphene electrodes provides very efficient electrostatic doping with Fermi energies of $1 \mathrm{eV}$ and a charge density of $8 \times 10^{13} \mathrm{~cm}^{-2}$. We show that the graphene supercapacitor yields more than $50 \%$ modulation between 0.1 and $1.4 \mathrm{THz}$ with operation voltages less than $3 \mathrm{~V}$. The low insertion losses, high modulation depth over a broad spectrum, and the simplicity of the device structure are the key attributes of graphene supercapacitors for THz applications. @ 2015 Optical Society of America
\end{abstract}

OCIS codes: (230.4110) Modulators; (160.4236) Nanomaterials.

http://dx.doi.org/10.1364/JOSAB.32.001861

\section{INTRODUCTION}

Optical modulators play a key role in optoelectronics and communication systems. Electro-optic, acousto-optic, and thermo-optic effects are well-established mechanisms to control intensity, phase, or polarization of light in the visible and nearinfrared frequencies. Efficient control of terahertz $(\mathrm{THz})$ waves, however, has been a challenge because of the lack of a $\mathrm{THz}$ active material [1]. Because of the high spatial resolution and nonionizing nature of $\mathrm{THz}$ waves, many emerging technologies require the ability to control and manipulate the intensity and phase of $\mathrm{THz}$ waves $[2,3]$. These technologies would benefit greatly from a $\mathrm{THz}$ modulator that has a simple device structure with efficient modulation. Tuning the bulk materials properties, such as dielectric constant and electrical conductivity, does not generate efficient reconfigurable $\mathrm{THz}$ components. Recent $\mathrm{THz}$ research is focused on controlling $\mathrm{THz}$ waves using highmobility, two-dimensional electron gas or high-mobility carriers on semiconductor surfaces $[1,4]$. Reverse bias voltage applied on Schottky contacts extends the depletion area under the metal-semiconductor interface that decreases the free carrier absorption. However, the metallic gate electrodes attenuate the $\mathrm{THz}$ waves and screen the surface charges that result in large insertion losses and limited modulation depth. Patterning the metallic gate electrodes as interconnected metamaterials eliminates some of these drawbacks. Chen $\mathrm{et} \mathrm{al}$. used interconnected split ring resonators as a gate electrode on GaAs substrates to control the depth of the depletion area under the gap of the resonator [5]. Various forms of metamaterial $\mathrm{THz}$ modulators have been demonstrated [6]. Requirements of the high-resolution photolithography process for large active device area and narrow spectral window hinder the realization of efficient $\mathrm{THz}$ modulators.

Graphene and other two-dimensional (2D) crystals provide new opportunities for $\mathrm{THz}$ technologies [7-11]. Atomic thickness and very large carrier mobility, together with the tunable optical properties, create a unique combination for active $\mathrm{THz}$ components. Since the thickness of $2 \mathrm{D}$ crystals $(\sim 0.3 \mathrm{~nm})$ is much thinner than the wavelengths of the $\mathrm{THz}$ waves, there is no bulk material that can effectively introduce large insertion losses. The $\mathrm{THz}$ response of the $2 \mathrm{D}$ crystals solely originates from high-mobility carriers which can be tuned by electrostatic doping. Particularly, graphene-based active $\mathrm{THz}$ devices have raised great interest in the last few years. The charge density in graphene can be tuned between $10^{12}$ and $10^{14} \mathrm{~cm}^{-2}$, which could yield efficient $\mathrm{THz}$ modulators with a wide dynamic range. Sensale-Rodriguez et al. reported a graphene-based $\mathrm{THz}$ modulator using a back-gated transistor structure. In their device, THz-transparent $\mathrm{Si}$ substrate operates as a back-gate electrode [12]. Using a graphene-dielectric-Si capacitor structure, the conductivity of graphene was modified by applying voltage between the Si back-gate and graphene. They further demonstrated individual and arrays of reflection and transmission-type $\mathrm{THz}$ modulators using back-gated device geometry [13-15]. The dynamic range of the back-gated device structure is limited by the electrical breakdown of the gate dielectric. Another approach is based on tuning the plasmon oscillations on 
structured graphene [16-18]. The frequency of plasmon oscillations on graphene ribbons can be tuned by the ribbon width and the charge density. Integrating graphene with metamaterials can yield another design parameter to improve the modulation depth; however, it narrows the spectral window. It has been predicted that grapheme-based modulators can create a modulation depth of $>90 \%$ [11]. However, because of the limited charge modulation with dielectric capacitors $\left(<10^{13} \mathrm{~cm}^{-2}\right)$, the full potential of graphene for $\mathrm{THz}$ technologies has yet to be achieved.

Recently, we have discovered that the supercapacitor geometry provides a very efficient device structure to control and manipulate optical properties of single-layer and multilayer graphene electrodes [19]. Using graphene supercapacitors, we fabricated optical modulators [19], electrochromic devices [20], tunable saturable absorbers [21], and radar absorbing surfaces [22] operating in visible, near-infrared, and microwave frequencies. Now, we would like to study the $\mathrm{THz}$ response of these graphene supercapacitors. In this paper, we demonstrate a simple, yet very efficient, broadband $\mathrm{THz}$ modulator using graphene supercapacitors.

The device consists of two large-area graphene electrodes transferred onto $\mathrm{THz}$ transparent substrates with ionic liquid electrolytes between them. Figure 1(a) shows the schematic drawing of the device layout. In this device geometry, there is no need for a metallic gate electrode which could introduce large insertion losses. Two graphene electrodes provide mutual gating. The bias voltage applied between graphene electrodes polarizes the electrolytes and yields efficient electrostatic doping on the graphene electrodes. The optical properties of graphene change with the doping [23-25].

Figure 1(b) shows the schematic band structure of doped graphene. There are two main electronic transitions that characterize the tunable optical response. The vertical interband electronic transition can be blocked via Pauli blocking when the Fermi energy is larger than the half of the photon energy. In supercapacitor configuration, the efficient gating of ionic liquid electrolyte and low electronic density of the states of graphene enable us to shift the Fermi energy in the order of one electron volt which modulates optical transmittance in the visible spectrum. In addition to the interband transitions, shifting the Fermi energy modifies the rate of intraband electronic transition because of the change in the electronic density of states [12]. These interband and intraband electronic transitions yield broadband absorption. We calculated the optical absorption of graphene using the transmission line model which provides an intuitive picture to understand the spectral response of our device [26]. The inset in Fig. 1(c) shows the equivalent transmission line model for single-layer graphene.

The graphene layer can be modeled as a thin film with thickness of $L(\sim 0.3 \mathrm{~nm})$ with a dynamic optical conductivity of

$$
\sigma(\omega)=\sigma_{\text {intra }}+\sigma_{\text {inter }}
$$

where $\sigma_{\text {intra }}$ and $\sigma_{\text {inter }}$ represent the optical conductivity because of intraband and interband transitions, respectively. The reflection $(R)$ and transmission $(T)$ coefficients can be written as [26]

$$
R=\frac{Z_{R}-1}{Z_{R}+1},
$$
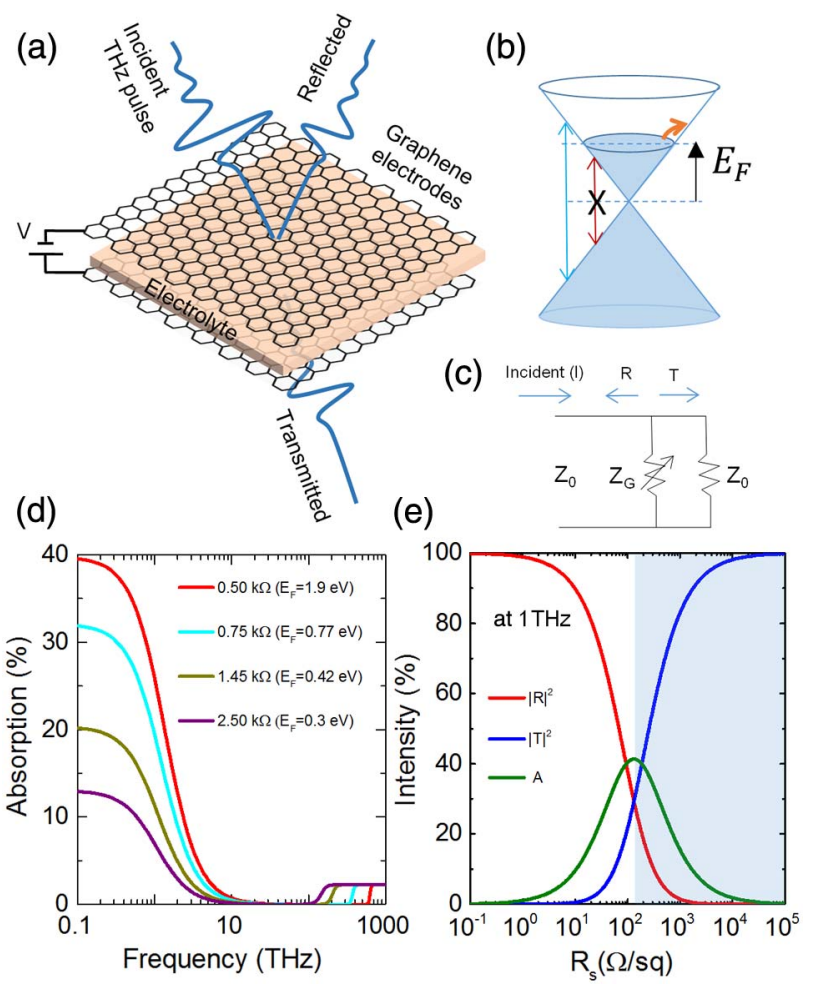

Fig. 1. (a) Schematic representation of the graphene supercapacitor used as a broadband $\mathrm{THz}$ modulator. The supercapacitor consists of ionic liquid electrolyte sandwiched between two large-area graphene electrodes. The charge density on graphene electrodes is modulated efficiently by an external voltage applied between the graphene electrodes. (b) Schematic band structure of electrostatically doped graphene electrodes. The arrows represent the interband and intraband electronic transitions. (c) Equivalent transmission line model of the graphene layer. (d) Calculated optical absorption of single-layer graphene plotted against the frequency for different doping levels. (e) Change of reflection, transmission, and absorption of graphene as a function of sheet resistance. The shaded area indicates the experimentally accessible sheet resistance for CVD graphene.

$$
\begin{gathered}
T=(1+R) \cos (k L)-j\left(\frac{Z}{Z_{0}}\right)(1-R) \sin (k L), \\
Z_{R}=\frac{1+j \frac{Z_{G}}{Z_{0}} \tan (k L)}{1+j \frac{Z_{0}}{Z_{G}} \tan (k L)}
\end{gathered}
$$

where $j$ is the imaginary number, and $Z_{0}$ represents characteristic impedance of the free space:

$$
Z_{0}=\sqrt{\frac{\mu_{0}}{\varepsilon_{0}}}=377 \Omega
$$

$Z_{G}$ is the characteristic impedance of the graphene layer:

$$
Z_{G}=\sqrt{\frac{\mu_{0}}{\left(\varepsilon^{\prime}-\sigma / j \omega\right)}}
$$

Here, $k$ is the complex wave vector of the $\mathrm{THz}$ wave. Figure 1(d) shows the calculated absorption of single-layer graphene,

$$
A=1-|R|^{2}-|T|^{2},
$$


from $\mathrm{THz}$ to visible spectra. In the $\mathrm{THz}$ regime, the absorption is because of intraband transition, where graphene behaves as a tunable Drude metal with a frequency-dependent optical conductivity of

$$
\sigma(\omega)_{\mathrm{intra}}=\frac{\sigma_{\mathrm{DC}}}{1+j \omega \tau},
$$

where $\sigma_{\mathrm{DC}}$ is the conductivity of graphene, $\omega$ is the frequency, and $\tau$ is the mean scattering time of graphene electrodes. For the calculation, we used a scattering time of $200 \mathrm{fs}$ which yields a cutoff frequency of $5 \mathrm{THz}$. In the optical frequencies, the intraband contribution diminishes because of a large momentum mismatch, resulting in a constant optical conductivity of $\mathrm{e}^{2} / 4$ because of the interband transitions. Figure 1(e) shows the variation of reflection, transmission, and absorption of graphene at $1 \mathrm{THz}$ with sheet resistance from $0.1 \Omega / \mathrm{sq}$ to $100 \mathrm{k} \Omega /$ sq. By tuning the sheet resistance of the graphene layer, the absorption can be altered from 40 down to 5\% [Fig. 1(e)]. The maximum $\mathrm{THz}$ absorption can be achieved when the characteristic impedance of graphene is matched to the free space impedance. The shaded area in Fig. 1(e) shows the experimentally accessible sheet resistance of CVD-grown graphene [27].

We fabricated the $\mathrm{THz}$ modulators using CVD-grown, largearea graphene layers. We used a chemical vapor deposition system to synthesize the large-area $(2 \times 2 \mathrm{~cm})$ graphene samples on ultra-smooth copper foil substrates (Mitsui Mining and Smelting Company, Ltd., B1-SBS, $100 \mathrm{~nm}$ surface roughness). The oxide layer on the foils was removed by a hydrogen flow during the annealing step. The graphene was synthesized at $1035^{\circ} \mathrm{C}$ using $40 \mathrm{sccm} \mathrm{CH}$ and $80 \mathrm{sccm} \mathrm{H}_{2}$ gases. After $20 \mathrm{~min}$ of growth time, the samples were cooled to the room temperature with the natural cooling rate of the furnace $\left(20^{\circ} \mathrm{C} / \mathrm{min}\right)$. After the growth, we coated the samples by drop casting thick Shipley 1813 photoresist which functions as a mechanical support for the transfer process. After etching the copper foils in an $\mathrm{FeCl}_{3}$ solution, the photoresist layer with graphene was transferred onto a quartz wafer. Reflowing the photoresist layer at $110^{\circ} \mathrm{C}$ on a quartz wafer results in conformal coating of graphene on the fused quartz surface. We attached two graphene-coated quartz wafers with a $25 \mu \mathrm{m}$ thick spacer, and filled the gap with ionic liquid electrolyte (Diethylmethyl(2-methoxyethyl) ammonium bis(trifluoromethylsulfonyl)imide, [deme][Tf2N]). The inset in Fig. 2(a) shows the fabricated $\mathrm{THz}$ modulator.

\section{OPTICAL AND ELECTRICAL CHARACTERIZATIONS OF GRAPHENE DEVICE}

Fermi energy $\left(E_{F}\right)$ of graphene is the primary parameter to describe the doping level. To extract the Fermi energy, we measured optical transmittance in the visible and near-infrared wavelength. The electrolyte gating yields Fermi energies on the order of $1 \mathrm{eV}$, which results in the blocking of interband transition in the near-infrared regime. The variation of the optical transmittance of the device with the bias voltage is shown in Fig. 2(a). The modulation of the transmittance shows a steplike function with a cutoff wavelength of $2 E_{F}$. As the bias voltage increases, the cutoff wavelength shifts to the higher energies. Since there are two graphene electrodes with slightly different
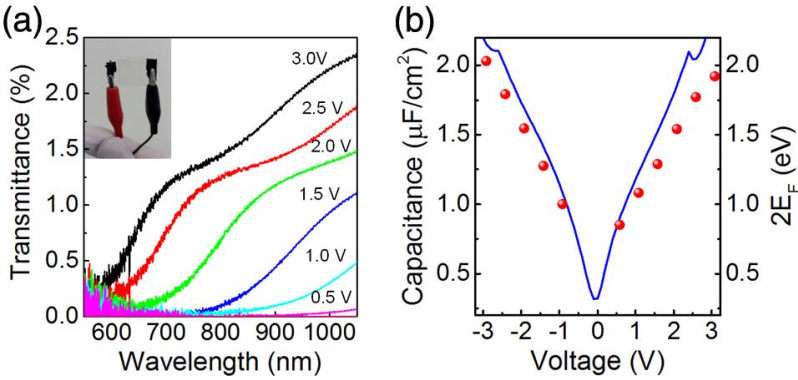

(c)

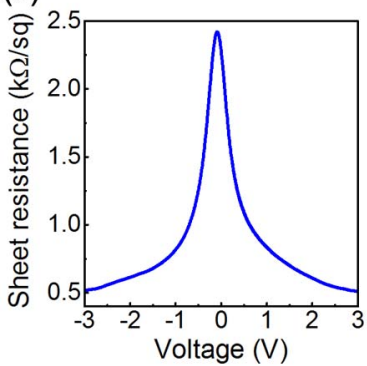

(d)

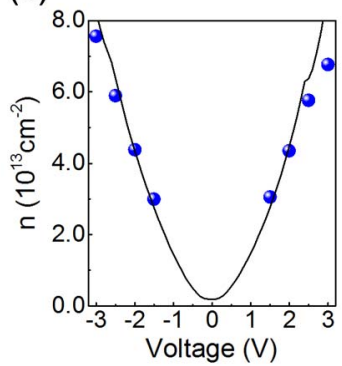

Fig. 2. (a) Modulation of the optical transmittance of the graphene supercapacitor in the visible spectra. (b) Variation of the capacitance of the devices and extracted Fermi energy of the graphene electrodes as a function bias voltage. (c) Variation of the resistance of the graphene electrodes with the bias voltage. (d) Calculated charge density is plotted against the bias voltage. The solid line is calculated from the capacitance, whereas the scattered points are calculated from the measured Fermi energy.

charge neutral points (Dirac point), we observe two steps in the transmittance spectra associated with each graphene layer.

Figure 2(b) shows the extracted Fermi energy of one graphene electrode as a function of bias voltage. The Fermi energy varies between 0.3 and $1.0 \mathrm{eV}$, as the bias voltage increases from 0.5 to $3 \mathrm{~V}$. The minimum Fermi energy associated with unintentional doping can be estimated to be $0.2 \mathrm{eV}$. The capacitance of the device provides more insight about the density of states of graphene electrodes. Figure 2(b) shows the variation of the capacitance as a function of bias voltage. To measure the voltage dependent capacitance, we superimposed $0.1 \mathrm{~V}$ alternating voltage and variable DC bias voltage. The variation of the capacitance between 0.3 and $2.2 \mu \mathrm{F}$ with the bias voltage is because of a change in the density of states as the Fermi energy shifts. The total capacitance of the device is limited by the low quantum capacitance $\left(C_{Q}\right)$ of the graphene electrodes which is directly related with the density of states of graphene electrodes. At the same time, the resistance of the graphene electrodes varies from $2.4 \mathrm{k} \Omega$ (at the Dirac point) down to $0.5 \mathrm{k} \Omega$ (at a bias voltage of $3 \mathrm{~V}$ ) [Fig. 2(c)]. Unlike a dielectric capacitor, in supercapacitors, both resistance of the electrodes and capacitance of the device change with the bias voltage. The calculated charge density on graphene is plotted against the bias voltage. At a bias voltage of $-3 \mathrm{~V}$, the charge density increases up to $8 \times 10^{13} \mathrm{~cm}^{-2}$ with Fermi energy of $1 \mathrm{eV}$. In Fig. 2(d), we plotted the total charge density as

$$
n=\sqrt{n_{0}^{2}+n_{g}^{2}},
$$


where $n_{0}=1.9 \times 10^{12} \mathrm{~cm}^{-2}$ is the estimated unintentional doping, and $n_{g}$ is the gate induced charges. To ensure the correct charge densities, we used measured Fermi energy and capacitance of the device. The solid line in Fig. 2(d) is calculated using the measured capacitance of the device, whereas the scattered point is calculated from the measured Fermi energy

$$
E_{F}=\hbar \nu_{F} \sqrt{n \pi},
$$

where $\nu_{F}$ is the Fermi velocity and $n$ is the charge density.

\section{BROADBAND THZ BEAM MODULATION USING GRAPHENE SUPERCAPACITORS}

Then we measured the $\mathrm{THz}$ response of the fabricated modulator. We used conventional time domain $\mathrm{THz}$ spectroscopy to measure the modulation of the intensity of transmitted $\mathrm{THz}$ signal. A Ti: $\mathrm{Al}_{2} \mathrm{O}_{3}$ mode-locked laser output with an average power of $200 \mathrm{~mW}$, a repetition rate of $75 \mathrm{MHz}$, and a pulse width of $15 \mathrm{fs}$ is used to drive the THz-TDS system. An ACbiased $(30 \mathrm{Vpp}, 1 \mathrm{KHz})$ photoconductive antenna is used for the generation of the $\mathrm{THz}$ pulses. The $\mathrm{THz}$ beam is collected and collimated by an off-axis parabolic mirror and then focused to a $\sim 8 \mathrm{~mm}$ spot size at the sample with the aid of a TPX lens $(\mathrm{D}=50 \mathrm{~mm}, \mathrm{~F} \# 2)$. After passing through the sample, the beam is collected by an identical lens and then focused by another off-axis parabolic mirror onto the detector crystal $(\langle 110\rangle$ $\mathrm{ZnTe}$ ) which allows it to be sampled using the electro-optic detection method. The entire $\mathrm{THz}$ beam path is enclosed in a box allowing it to be purged with dry nitrogen during the measurements. Figure 3(a) shows the time-varying electric field of the $\mathrm{THz}$ pulse recorded with the THz-TDS system. To
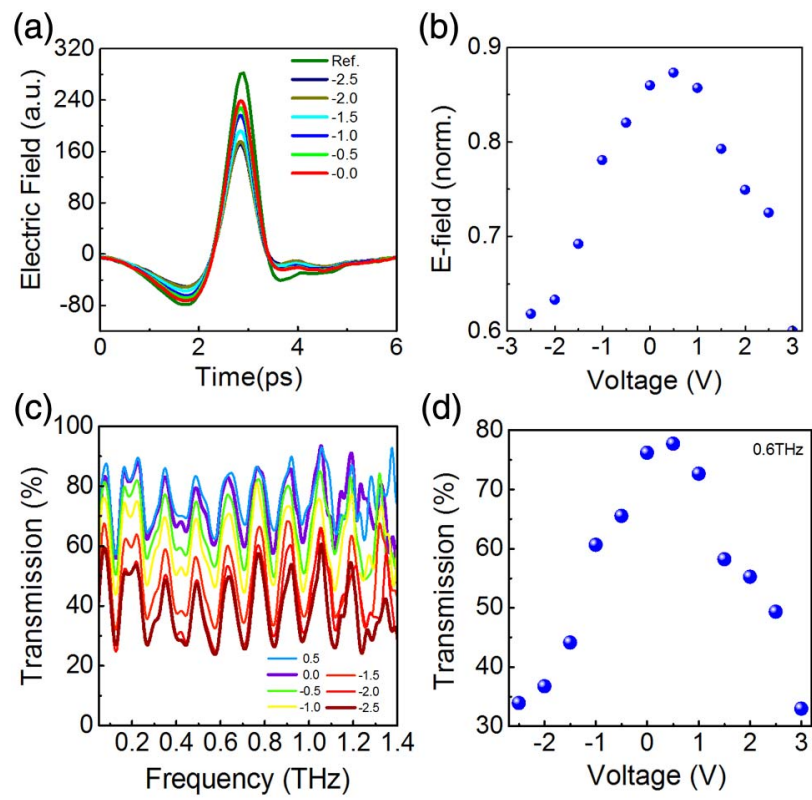

Fig. 3. (a) Time-varying electric field of the $\mathrm{THz}$ pulse at various voltage bias between 0 and $-2.5 \mathrm{~V}$. The reference $\mathrm{THz}$ signal is measured without the graphene supercapacitor. (b) Modulation of the maximum electric field as a function of bias voltage. (c) Spectrum of the transmitted $\mathrm{THz}$ pulse obtained after Fourier transform of the transmitted signal and normalization with the reference signal. (d) Modulation of signal at $0.6 \mathrm{THz}$. remove the substrate effects, we fabricated an identical test device without the graphene electrodes. The reference signal is recorded using this test device. We measured the transmitted $\mathrm{THz}$-pulse through the graphene supercapacitor at different bias voltages. Figure 3(b) shows the modulation of the maximum electric field which is normalized by the reference signal. The normalized transmitted electric field decreases with the bias voltage from 0.87 down to 0.61 at a bias voltage of $-2.5 \mathrm{~V}$. The asymmetry in the modulation is because of the hysteresis caused by the shift in the Dirac point. Small leakage current induces unintentional doping on graphene which shifts the Dirac point. By performing Fourier analysis, we obtained the spectral amplitude and phase of the transmitted signal [Fig. 3(c)]. We observed clear Fabry-Perot resonances with a period of $\sim 0.12 \mathrm{THz}$. These Fabry-Perot resonances originate from the parallel quartz surfaces. The amplitude of the transmitted signal is modulated by around $55 \%$ in broad spectra from 0.1 to $1.4 \mathrm{THz}$ which is limited by the transparency of the quartz substrate. The modulation of the transmittance at $0.6 \mathrm{THz}$ is shown in Fig. 3(d).

Because of the material cost and requirement of highresolution lithography, fabrication of large-area $\mathrm{THz}$ modulators has been a challenge. The simplicity of our device structure allows us to fabricate a very large-area broadband $\mathrm{THz}$ modulator. To demonstrate the promise of our approach, we synthesized large-area $7 \times 7 \mathrm{~cm}$ graphene, and then we laminated flexible $125 \mu \mathrm{m}$ thick PVC (polyvinyl chloride) substrate on a graphene-coated surface of the foil at $120^{\circ} \mathrm{C}$. Etching the copper foil yields large-area crack-free graphene on a $\mathrm{THz}$ transparent and flexible PVC substrate. The PVC substrate is $\mathrm{THz}$ transparent between 0.1 and $1.7 \mathrm{THz}$. Figure 4(a) shows the fabricated large-area $\mathrm{THz}$ modulator. During the etching process, we covered the edges of the sample and left thin copper strips which function as metal electrodes. First, we measured the variation of the capacitance of the device with the bias voltage [Fig. 4(b)]. The total capacitance varies between $70 \mu \mathrm{F}$ at $-3 \mathrm{~V}$ down to $10 \mu \mathrm{F}$ at $0 \mathrm{~V}$. This corresponds to capacitance per unit area of $3 \mu \mathrm{F} / \mathrm{cm}^{2}$. As the area of the device increases, the total capacitance of the modulator increases which limits the cutoff frequency. To quantify the cutoff frequency, we fabricated various $\mathrm{THz}$ modulators and measured their frequency-dependent capacitance [Fig. 4(c)]. Figure 4(d) shows the variation of the extracted cutoff frequency with the device area. We observed that the cutoff frequency is inversely proportional with the device area. Figure 4(e) shows the modulation of the $\mathrm{THz}$ signal. The performance of the device is similar to the one based on a quartz substrate with lower insertion loss. Unlike the quartz, the two flexible PVC substrates are not flat enough to support Fabry-Perot resonances Fig. 4(f).

Insertion loss and modulation depth are the two important parameters for $\mathrm{THz}$ modulators. In Fig. 5(a) we show the intrinsic insertion loss resulting from the two graphene electrodes and the total insertion loss of the graphene supercapacitor, including a quartz substrate for a frequency range from 0.1 to $1.4 \mathrm{THz}$. The intrinsic insertion loss is around $0.7 \mathrm{~dB}$. In Fig. 5(b), we compare our device performance with the $\mathrm{THz}$ modulators reported in the literature [6,28-31]. Our device shows a maximum modulation of $62 \%$ and an intrinsic loss 
(a)

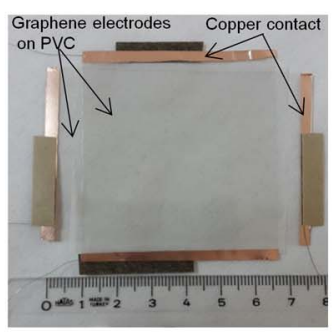

(b)

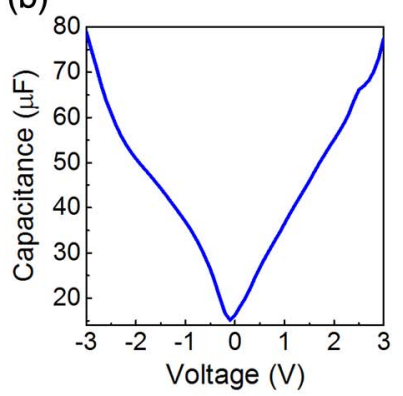

(c)

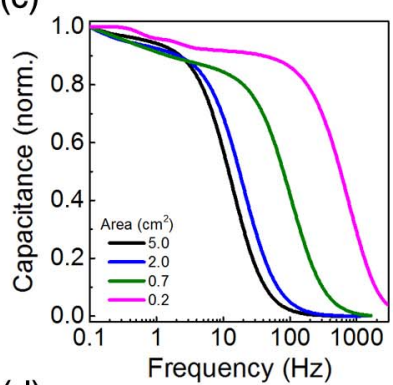

(d)

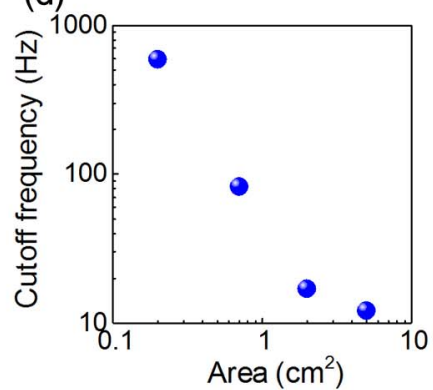

(e)

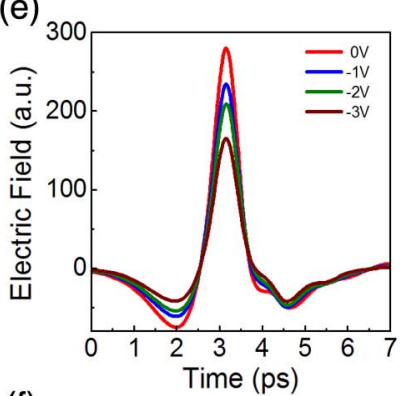

(f)

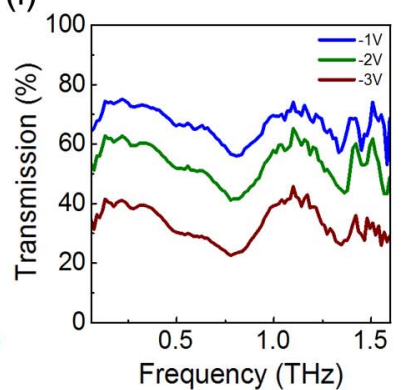

Fig. 4. (a) Picture of the large-area $(7 \times 7 \mathrm{~cm}) \mathrm{THz}$ modulator. (b) Variation of the capacitance of the modulator as a function of bias voltage. (c) Capacitance of the modulator at different sizes. (d) Extracted cutoff frequency as a function of device size. (e) Time-varying electric field of the THz pulse at various voltage bias between 0 and -3 V. (f) Spectrum of the transmitted THz signal obtained after Fourier transformation of the recorded signal and normalization with the signal at a bias voltage of $0 \mathrm{~V}$.
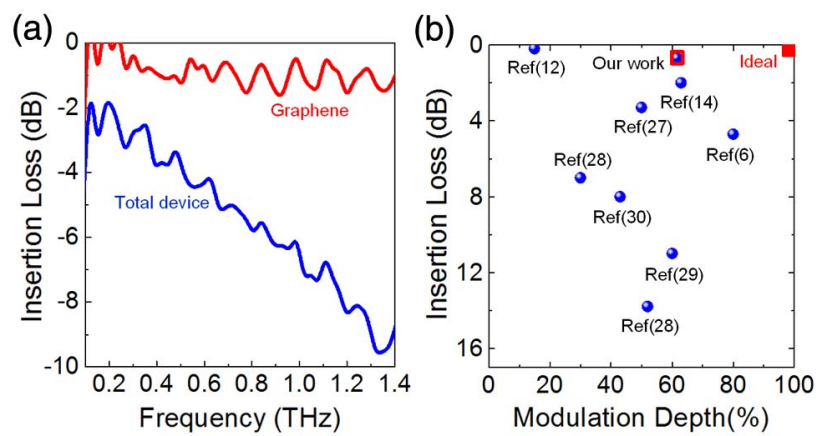

Fig. 5. (a) Insertion loss of the total device and the graphene electrodes plotted against the frequency. (b) Comparative graph showing the modulation depth and intrinsic insertion loss of graphene modulators.

of $0.7 \mathrm{~dB}$. This combination provides a clear advantage over the previous modulators. In addition, our device provides the largest reported spectral window.

\section{CONCLUSIONS}

In conclusion, we demonstrated a $\mathrm{THz}$ intensity modulator using large-area graphene supercapacitors. We fabricated the graphene supercapacitors using CVD-grown graphene layers and ionic liquid electrolyte. The supercapacitor structure provides efficient mutual gating between two graphene electrodes. With this simple device structure, we were able to modulate the intensity of $\mathrm{THz}$ waves with a modulation depth of $50 \%$ between 0.1 and $1.4 \mathrm{THz}$ with operation voltages less than $3 \mathrm{~V}$. This device can operate as a reflection type modulator as well.
Furthermore, we demonstrated a very large area $7 \times 7 \mathrm{~cm} \mathrm{THz}$ modulator on a flexible substrate. The low insertion loss, the simplicity of the device structure, and polarization independent device performance are the key attributes of graphene supercapacitors for $\mathrm{THz}$ applications. Another interesting aspect of this device is that the graphene supercapacitor is active in a very broad spectrum, ranging from visible to $\mathrm{THz}$ frequencies. The slow response time and the requirement of liquid electrolyte are the drawbacks of our approach.

Funding. Türkiye Bilimsel ve Teknolojik Arastirma Kurumu (Scientific and Technological Research Council of Turkey) (114F379).

\section{REFERENCES}

1. M. Rahm, J. S. Li, and W. J. Padilla, "THz wave modulators: a brief review on different modulation techniques," J. Infrared Millim. Terahertz Waves 34, 1-27 (2013).

2. C. Sirtori, S. Barbieri, and R. Colombelli, "Wave engineering with $\mathrm{THz}$ quantum cascade lasers," Nat. Photonics 7, 691-701 (2013).

3. M. Tonouchi, "Cutting-edge terahertz technology," Nat. Photonics 1, 97-105 (2007).

4. T. Kleine-Ostmann, P. Dawson, K. Pierz, G. Hein, and M. Koch, "Room-temperature operation of an electrically driven terahertz modulator," Appl. Phys. Lett. 84, 3555-3557 (2004).

5. H. T. Chen, J. F. O'Hara, and A. J. Taylor, "Active terahertz metamaterials," Opt. Spectrosc. 108, 834-840 (2010).

6. H. T. Chen, W. J. Padilla, M. J. Cich, A. K. Azad, R. D. Averitt, and A. J. Taylor, "A metamaterial solid-state terahertz phase modulator," Nat. Photonics 3, 148-151 (2009).

7. P. Tassin, T. Koschny, and C. M. Soukoulis, "Graphene for terahertz applications," Science 341, 620-621 (2013).

8. B. Sensale-Rodriguez, R. S. Yan, L. Liu, D. Jena, and H. G. Xing, "Graphene for reconfigurable terahertz optoelectronics," Proc. IEEE 101, 1705-1716 (2013). 
9. R. Degl'Innocenti, D. S. Jessop, Y. D. Shah, J. Sibik, J. A. Zeitler, P. R. Kidambi, S. Hofmann, H. E. Beere, and D. A. Ritchie, "Terahertz optical modulator based on metamaterial split-ring resonators and graphene," Opt. Eng. 53, 057108 (2014).

10. S. H. Lee, M. Choi, T. T. Kim, S. Lee, M. Liu, X. Yin, H. K. Choi, S. S. Lee, C. G. Choi, S. Y. Choi, X. Zhang, and B. Min, "Switching terahertz waves with gate-controlled active graphene metamaterials," Nat. Mater. 11, 936-941 (2012).

11. B. Sensale-Rodriguez, T. Fang, R. S. Yan, M. M. Kelly, D. Jena, L. Liu, and H. L. Xing, "Unique prospects for graphene-based terahertz modulators," Appl. Phys. Lett. 99, 113104 (2011).

12. B. Sensale-Rodriguez, R. Yan, M. M. Kelly, T. Fang, K. Tahy, W. S. Hwang, D. Jena, L. Liu, and H. G. Xing, "Broadband graphene terahertz modulators enabled by intraband transitions," Nat. Commun. 3, 780-786 (2012).

13. J. S. Gomez-Diaz, C. Moldovan, S. Capdevilla, J. Romeu, L. S. Bernard, A. Magrez, A. M. Ionescu, and J. Perruisseau-Carrier, "Self-biased reconfigurable graphene stacks for terahertz plasmonics," arXiv:1405.3320 (2014).

14. B. Sensale-Rodriguez, R. S. Yan, S. Rafique, M. D. Zhu, W. Li, X. L. Liang, D. Gundlach, V. Protasenko, M. M. Kelly, D. Jena, L. Liu, and H. G. Xing, "Extraordinary control of terahertz beam reflectance in graphene electro-absorption modulators," Nano Lett. 12, 4518-4522 (2012).

15. B. Sensale-Rodriguez, S. Rafique, R. S. Yan, M. D. Zhu, V. Protasenko, D. Jena, L. Liu, and H. G. Xing, "Terahertz imaging employing graphene modulator arrays," Opt. Express 21, 2324-2330 (2013).

16. L. Ju, B. S. Geng, J. Horng, C. Girit, M. Martin, Z. Hao, H. A. Bechtel, X. G. Liang, A. Zettl, Y. R. Shen, and F. Wang, "Graphene plasmonics for tunable terahertz metamaterials," Nat. Nanotechnol. 6, 630-634 (2011).

17. M. Jablan, H. Buljan, and M. Soljacic, "Plasmonics in graphene at infrared frequencies," Phys. Rev. B 80, 245435 (2009).

18. H. Min and A. H. MacDonald, "Origin of universal optical conductivity and optical stacking sequence identification in multilayer graphene," Phys. Rev. Lett. 103, 067402 (2009).

19. E. O. Polat and C. Kocabas, "Broadband optical modulators based on graphene supercapacitors," Nano Lett. 13, 5851-5857 (2013).
20. E. O. Polat, O. Balci, and C. Kocabas, "Graphene based flexible electrochromic devices," Sci. Rep. 4, 6484 (2014).

21. I. Baylam, M. N. Cizmeciyan, S. Ozharar, E. O. Polat, C. Kocabas, and A. Sennaroglu, "Femtosecond pulse generation with voltagecontrolled graphene saturable absorber," Opt. Lett. 39, 5180-5183 (2014).

22. O. Balci, E. O. Polat, N. Kakenov, and C. Kocabas, "Grapheneenabled electrically switchable radar-absorbing surfaces," Nat. Commun. 6, 6628 (2015)

23. Z. Q. Li, E. A. Henriksen, Z. Jiang, Z. Hao, M. C. Martin, P. Kim, H. L. Stormer, and D. N. Basov, "Dirac charge dynamics in graphene by infrared spectroscopy," Nat. Phys. 4, 532-535 (2008).

24. F. Wang, Y. B. Zhang, C. S. Tian, C. Girit, A. Zettl, M. Crommie, and Y. R. Shen, "Gate-variable optical transitions in graphene," Science 320, 206-209 (2008).

25. K. F. Mak, L. Ju, F. Wang, and T. F. Heinz, "Optical spectroscopy of graphene: from the far infrared to the ultraviolet," Solid State Commun. 152, 1341-1349 (2012).

26. H. Bosman, Y. Y. Lau, and R. M. Gilgenbach, "Microwave absorption on a thin film," Appl. Phys. Lett. 82, 1353-1355 (2003).

27. S. Bae, H. Kim, Y. Lee, X. F. Xu, J. S. Park, Y. Zheng, J. Balakrishnan, T. Lei, H. R. Kim, Y. I. Song, Y. J. Kim, K. S. Kim, B. Ozyilmaz, J. H. Ahn, B. H. Hong, and S. lijima, "Roll-to-roll production of 30-inch graphene films for transparent electrodes," Nat. Nanotechnol. 5, 574-578 (2010).

28. H. T. Chen, W. J. Padilla, J. M. O. Zide, A. C. Gossard, A. J. Taylor, and R. D. Averitt, "Active terahertz metamaterial devices," Nature 444, 597-600 (2006).

29. H. T. Chen, H. Lu, A. K. Azad, R. D. Averitt, A. C. Gossard, S. A. Trugman, J. F. O'Hara, and A. J. Taylor, "Electronic control of extraordinary terahertz transmission through subwavelength metal hole arrays," Opt. Express 16, 7641-7648 (2008).

30. O. Paul, C. Imhof, B. Lagel, S. Wolff, J. Heinrich, S. Hofling, A. Forchel, R. Zengerle, R. Beigang, and M. Rahm, "Polarizationindependent active metamaterial for high-frequency terahertz modulation," Opt. Express 17, 819-827 (2009).

31. D. Shrekenhamer, S. Rout, A. C. Strikwerda, C. Bingham, R. D. Averitt, S. Sonkusale, and W. J. Padilla, "High speed terahertz modulation from metamaterials with embedded high electron mobility transistors," Opt. Express 19, 9968-9975 (2011). 